\title{
Tabelle der Abkürzungen
}

*) bezeichnet Abkürzungen, die nicht in der von der IUPAC bzw. IUB empfohlenen Abkürzungstabelle enthalten sind.

1. Symbole für monomere Einheiten in Makromolekülen oder in phosphorylierten Verbindungen

Symbol

A

Ala

Arg

Asp

*Asp- $\mathrm{NH}_{2}$

C

Cys oder Cys

Cys

d

dRib

Fru

Gal

Glc

G

GlcA

GlcN

GlcNAc

GlcUA

Glu

*Glu- $\mathrm{NH}_{2}$

Gly

His

$\mathrm{Hyl}$

Hyp monomere Einheit

Adenosin

Alanin

Arginin

Asparaginsäure

Asparagin

Cytidin

Cystin (halb)

Cystein

„desoxy" in Kohlenhydraten und Nucleotiden

2-Desoxyribose

Fructose

Galaktose

Glucose (auch G, wenn keine Verwechslung

mit Guanosin möglich ist)

Guanosin

Gluconsäure

Glucosamin

$\mathrm{N}$-Acetylglucosamin

Glucuronsảure

Glutaminsäure

Glutamin

Glycin (bzw. Glykokoll)

Histidin

Hydroxylysin

Hydroxyprolin 


\begin{tabular}{|c|c|}
\hline Symbol & monomere Einheit \\
\hline I & Inosin \\
\hline Ile & Isoleucin \\
\hline Leu & Leucin \\
\hline Lys & Lysin \\
\hline Man & Mannose \\
\hline Met & Methionin \\
\hline NANA & N-Acetylneuraminsäure \\
\hline Orn & Ornithin \\
\hline$*(\mathbb{P}$ & anorganisches Phosphat \\
\hline$* \mathscr{P}-$ & Phosphoryl-(Esterphosphat) \\
\hline$* \mathscr{P}-\mathbb{P}$ & Pyrophosphat (Diphosphat) \\
\hline *(P)- $(\mathbb{P})$ & Pyrophosphoryl-(Diphosphatester) \\
\hline Phe & Phenylalanin \\
\hline Pro & Prolin \\
\hline Rib & Ribose \\
\hline Ser & Serin \\
\hline Thr & Threonin \\
\hline $\operatorname{Trp}$ & Tryptophan (auch Try) \\
\hline $\mathrm{T}$ & Thymidin \\
\hline $\mathrm{dT}$ & Desoxyribosylthymin \\
\hline Tyr & Tyrosin \\
\hline $\mathrm{U}$ & Uridin \\
\hline Val & Valin \\
\hline
\end{tabular}

2. Abkürzungen für halbsystematische oder Trivialnamen

Acetyl-CoA

$\mathrm{ACTH}$

ADP

AMP

ATP

CDP

CMP

$\mathrm{CoA}$

*-CoA

*- $\overline{\mathrm{CoA} \mid}$

CTP
Acetylcoenzym A

Adrenocorticotropin, adrenocorticotropes Hormon Adenosin-5'-diphosphat

Adenosin- $5^{\prime}$-phosphat

Adenosin-5'-triphosphat

Cytidin-5'-diphosphat

Cytidin- $5^{\prime}$-phosphat

freies Coenzym A

Coenzym $\mathrm{A}$ in Thioesterbindung

Coenzym $A$ in Thioesterbindung (in Formeln)

Cytidin-5'-triphosphat 
DNA

DOPA

FAD

FMN

GDP

GMP

GSH

GSSG

GTP

*[H]

HHL

$\mathrm{Hb}, \mathrm{HbCO}, \mathrm{HbO}_{2}$

IDP

IMP

ITP

*I. P.

MSH

NAD

*NADH

NADP

*NADPH

NMN

NNR

PAPS

RNA

STH

UDP

UDPG

UMP

UTP
Desoxyribonucleinsäure

Dihydroxy-phenylalanin

Flavinadenindinucleotid

Riboflavin-5'-phosphat

Guanosin-5'-diphosphat

Guanosin-5'-phosphat

Glutathion

oxydiertes Glutathion

Guanosin-5'-triphosphat

$\mathrm{H}^{+}+\mathrm{e}^{-}$

Hypophysenhinterlappen

Hämoglobin, Kohlenmonoxid-Hämoglobin, Oxyhämoglobin

Inosin-5'-diphosphat

Inosin-5'-phosphat

Inosin-5'-triphosphat

Isoelektrischer Punkt

Melanozyten-stimulierendes Hormon

$\mathrm{NAD}^{+}$, Nicotinamidadenindinucleotid

(früher DPN)

$\mathrm{NADH}+\mathrm{H}^{+}$, reduziertes NAD

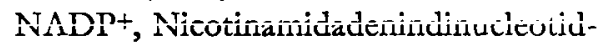

phosphat (früher TPN)

$\mathrm{NADPH}+\mathrm{H}^{+}$, reduziertes NADP

Nicotinamidmononucleotid

Nebennierenrinde

3'-Phosphoadenosin-5'-phosphosulfat

Ribonucleinsäure

Somatotropes Hormon

Uridin-5'-diphosphat

Uridin-5'-diphosphat-glucose

Uridin-5'-phosphat

Uridin-5'-triphosphat

3. Symbole in der Enzymkinetik

Geschwindigkeit einer enzymatischen Reaktion

Geschwindigkeit einer enzymatischen Reaktion bei Substratsättigung (= Maximalgeschwindigkeit) 
$\mathrm{K}_{\mathrm{m}}$

$K_{s}$

$\mathrm{K}_{\mathrm{i}}$

$\mathrm{k}_{+\mathrm{n}}, \mathrm{k}_{-\mathrm{n}}$

$\mathrm{U}$

kat
MICHAELIS-Konstante. Substratkonzentration, bei $\operatorname{der} \mathrm{v}=\mathrm{V} / 2$ ist.

Substratkonstante. Geschwindigkeits-(Dissoziations-) konstante der Reaktion $\mathrm{E}+\mathrm{S} \rightleftarrows \mathrm{ES}$

Inhibitorkonstante. Geschwindigkeits-(Dissoziations-) konstante der Reaktion $\mathrm{E}+\mathrm{I} \rightleftarrows \mathrm{EI}$

Geschwindigkeitskonstanten der Hin- und Rückreaktion beim n.Schritt einer enzymatischen Reaktion

Enzymeinheit. Eine Einheit ist die Menge eines Enzyms, welche die Umwandlung von $1 \mu \mathrm{Mol}$ Substrat/ Min. unter definierten Bedingungen katalysiert.

Neue Enzymeinheit (Katal.) Ein Katal ist die Menge eines Enzyms, welche die Umwandlung von einem Mol Substrat/sec unter definierten Bedingungen katalysiert.

\section{Allgemeine Abkürzungen und Symbole}

$\AA$

Abb.

Atm

$e^{-}$

$\mathrm{g}$

Kap.

Min.

Mol.-Gew.

Std.

Stdn.

Tab.

UV

z. 'T.

$\varnothing$

$>$

$<$

$\sim$
Angström-Einheit $\left(1 \AA=10^{-10} \mathrm{~m}\right)$

Abbildung

Atmosphäre (1/760 Atm $=1$ Torr $=1 \mathrm{~mm} \mathrm{Hg})$

Elektron

Erdbeschleunigung (Fallbeschleunigung $=$ $9,81 \mathrm{~m} / \mathrm{sec}^{2}$ )

Kapitel

Minute

Molekulargewicht

Stunde

Stunden

Tabelle

Ultraviolett

zum Teil

Durchmesser

größer als

kleiner als

„energiereiche“ Bindung

Weitere Abkürzungen und Symbole im Text. 